\section{This week in therapeutics}

Target/marker/
Indication pathway
Infectious disease

Bacterial

Unknown

infection;

infectious disease

\section{Summary}

In vitro and mouse studies identified

5-nitroimidazole (5-NI) derivatives that could help treat drug-resistant bacterial and protozoan infections. In growth and survival assays for Giardia lamblia, Trichomonas vaginalis, Helicobacter pylori and Clostridium difficile, 44 of the 378 tested 5-NI derivatives were more effective against all 4 organisms than the parent compound metronidazole. In these assays, the 5-NI derivatives showed antimicrobial activity against multiple clinical isolates, including those resistant to metronidazole. In mouse models of giardiasis, 7 of 16 tested 5-NI derivatives showed greater antimicrobial activity against the parasite than metronidazole. Next steps include selecting specific indications for testing individual compounds.

SciBX 6(43); doi:10.1038/scibx.2013.1225 Published online Nov. 7, 2013

\section{Licensing status}

Patent pending; available for licensing

\title{
THE DISTILLERY
}

\section{Publication and contact information}

Miyamoto, Y. et al. Proc. Natl. Acad. Sci. USA; published online

Oct. 7, 2013;

doi:10.1073/pnas.1302664110

Contact: Lars Eckmann, University of California, San Diego, La Jolla, Calif. e-mail:

leckmann@ucsd.edu 